\title{
Standardless EDS Composition Analysis using Quantitative Annular Dark-Field Imaging
}

\author{
J. Houston Dycus ${ }^{1}$, Weizong $\mathrm{Xu}^{1}$ and James M. LeBeau ${ }^{1}$ \\ 1. Department of Materials Science and Engineering, North Carolina State University, Raleigh, North \\ Carolina 27695, USA
}

Breakthroughs in energy dispersive X-ray detectors have greatly improved the signal-to-noise ratio of spatial mapping, leading to new interest for quantitative atomic scale elemental analysis. The most common method for EDS composition analysis in TEM is the Cliff-Lorimer ratio where the composition is directly related to the X-ray peak intensities and the Cliff-Lorimer factor, $K$, through: $\frac{C A}{C B}=k_{A B} * \frac{I A}{I B}$ [1]. Although the method makes for a simple way to extract quantitative information, accurately calibrating $K$ factors can be difficult. Another method for compositional EDS analysis is using the $\zeta$ factor approach where the characteristic X-ray intensities are related to the mass-thickness and composition [2]. For both quantitative methods, samples are tilted to minimize channeling conditions; however, orientation along a low-order zone axis, resulting in strong channeling, is required for atomic scale analysis. The necessity for on-zone acquisition has resulted in studies to account for channeling conditions to improve the quantitative capabilities of atomic scale EDS mapping [3]. An alternative method for determining composition is quantitative STEM (QSTEM) using the annular dark-field intensity [4-5]. In this approach, intensity is placed on an absolute scale and multislice image simulations are performed at varying composition and thickness values. Importantly, a standard with known composition is not needed for composition analysis using QSTEM and images are typically acquired at strong channeling conditions.

In this presentation, we will show that $\mathrm{K}$ and $\zeta$-factors can be accurately calibrated for samples of unknown composition using QSTEM. Using a disordered ternary AlGaN alloy grown with layers of varying composition, Figure 1A, we first determine the composition of one layer using QSTEM to calibrate the Cliff-Lorimer ratio, Figure 1B. Afterwards, this ratio can be applied the other layers to determine composition. Figure 2 shows the fractional occupancy of $\mathrm{Ga}$ in the $\mathrm{Ga} / \mathrm{Al}$ site for each set of layers in the sample. Importantly, the composition of each layer was unknown prior to analysis. Comparing the composition values for the QSTEM approach and the standard K-factor approach, the maximum absolute variation is $5.2 \%$ for any layer. We will then discuss how this method is beneficial for thicker samples and samples oriented to channeling conditions where many factors plague composition analysis for conventional methods. To check the accuracy of the method, we compare compositional results with the nominally intended growth concentrations. With respect to the standard Cliff-Lorimer and $\zeta$-factor approaches, we will compare the accuracy of our newly presented method. This approach enables composition analysis without the need for a calibration reference. 


\section{References:}

[1] D. J. Lichtenwalner et al, Applied Physics Letters, 105(18) (2014).

[1] G. Cliff and G.W. Lorimer, Journal of Microscopy 103(2) (1975), p. 203-207.

[2] M. Watanabe, and D. B. Williams, Journal of Microscopy 221(2) (2006), p. 89-109.

[3] Z. Chen et al, Ultramicroscopy 168 (2016), p. 7-16.

[4] J. M. LeBeau et al, Physical Review Letters 100(20) (2008), p. 206101.

[5] A. Rosenauer et al. Ultramicroscopy 109(9) (2009), p. 1171-1182.

[9] JHD, WX and JML acknowledge the Analytical Instrumentation Facility (AIF) at North Carolina State University. JHD acknowledges support by the National Science Foundation Graduate Research Fellowship (Grant DGE-1252376).

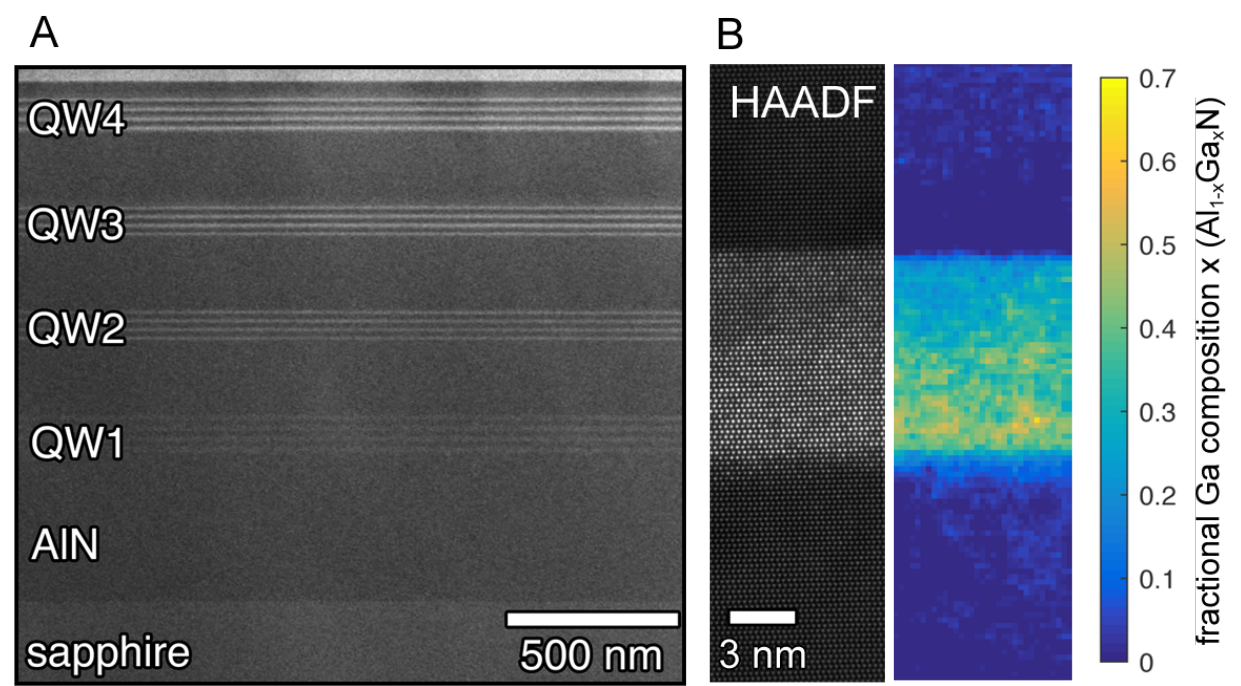

Figure 1: Quantitative STEM imaging for an $\mathrm{Al}_{1-\mathrm{x}} \mathrm{Ga}_{\mathrm{x}} \mathrm{N}$ film grown on sapphire. (A) Image of an overview of the film. (B) Quantitative STEM image from on layer in QW2 (left) beside the composition map (right).

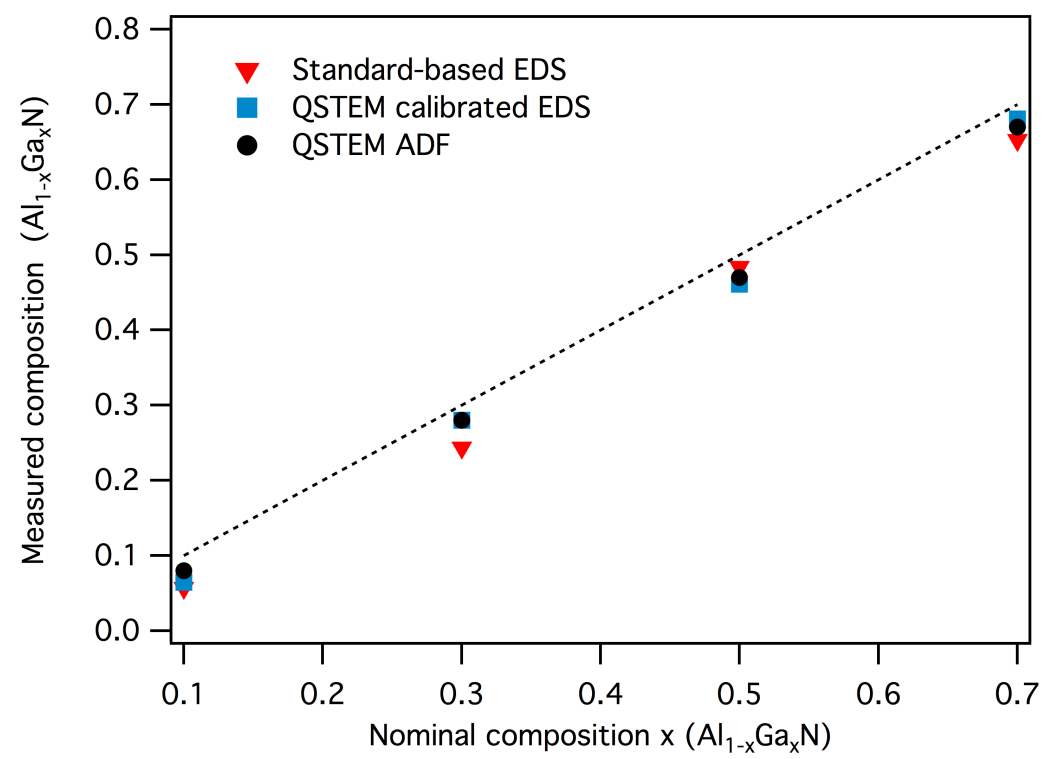

Figure 2: Plot of the average Ga content in the QWs as a function of the nominally intended composition for the standard K-factor approach and QSTEM calibrated methods. 